Meklenborg, Lohndal \& Østby (eds) Syntax, semantics and acquisition: In honor of Hans Petter Helland, Oslo Studies in Language 12(1), 2021. 105-123. (ISSN 1890-9639/ISBN 978-82-91398-12-9)

http://wWw.journals.uio.no/osla

\title{
LES COORDONNANTS ET L'INVERSION DU SUJET EN FRANÇAIS ANCIEN
}

\author{
PIERRE LARRIVÉE \\ Université de Caen Normandie
}

RÉSUMÉ

Le point de départ de ce travail est le constat que le français ancien, qui contient des constructions à verbe en seconde position avec sujet postposé comme c'est par exemple le cas en norvégien, a également des sujets postposés à un verbe précédé d'un coordonnant, structure inexistante en norvégien. La construction à inversion du sujet avec coordonnant initial en français ancien est réévaluée ici. Cette construction a tendance à être analysée par les études existantes comme étant une coordination d'un verbe en position syntaxiquement basse suivie du sujet in situ, et non pas comme une structure à verbe second avec le verbe en position haute. L'analyse est fondée sur l'absence de postposition de sujets clitiques dans les données analysées. Ces données sont cependant essentiellement littéraires, et au regard de l'attestation de sujets clitiques dans d'autres langues romanes comme l'italien, des données alternatives du français sont examinées. Ces données confirment que la construction ne relève pas du verbe en seconde position, parce que le verbe ne figure pas en position syntaxique haute. Elle illustre plutôt une coordination asymétrique, ce qui est courant dans l'ancienne langue, expliquant les divergences d'avec le norvégien et le français actuel.

\section{[1] INTRODUCTION}

Proposer un travail pour souligner la contribution d'Hans Petter Helland n'est pas tâche facile. Il faut honorer le syntacticien qui est une figure de référence de la grammaire du français contemporain (Helland 2006). Il faut rendre hommage au comparatiste qui sait faire voir à l'éclairage de l'autre langue les lumières et les points d'ombre du norvégien et du français. Et sa sagacité scientifique ne saurait se voir offrir le traitement d'une quelconque question controuvée.

C'est donc une question classique que ce travail explore. Y sont considérées les configurations avec inversion du sujet introduites par un coordonnant en français ancien. Elles peuvent être illustrées par les exemples suivants tirés du corpus FLAN+ de textes légaux normands. 
(1) a. il fu jugié en l'eschequier que cil jugemenz estoit faus, il fut jugé en le parlement que ce jugment était faux et ot Aales la sèsine (1207. Arrêt de l'échiquier de Normandie) et eut Aales la saisine " et Aales en eut la possession»

b. Et ne n'ot le Roy noient (1279. Enquête Pont Audemer) et ni n'eut le roi rien 'Et le Roy n'eut rien'

c. et dist ledit Pierre que ... (1355. Plès Mustel) et dit ledit Pierre queZ «Et ledit Pierre dit que ...»

Ces illustrations semblent relever de l'ordre des mots avec verbe en seconde position (V2). Cette configuration implique un verbe qui se retrouve dans une position syntaxique haute. Dans cette position, le verbe est précédé d'un constituant, également en position syntaxique haute, dite par Skårup « position du fondement » (1975). Si ce constituant n'est pas le sujet, ce dernier se trouve reporté en position post-verbale. Relativement bien représentée en ancien français, cette construction a graduellement disparu, alors qu'elle a subsisté dans les langues germaniques. Or, de façon intéressante, dans une langue germanique comme le norvégien actuel où V2 est opératoire, des séquences V2 à coordonnant initial sont inattestées, ainsi que le montre la dernière de ces élicitations.
a. Læreren
sa
det.
Enseignant-DEF dire-PRETERIT-3SG cela «L'enseignant l'a dit. »
b. Og læreren sa det. Et enseignant-DEF dire-Preterit-3SG cela. «Et l'enseignant l'a dit. »
c. *ogsa læreren det
Et dire-Preterit-3SG enseignant-DEF cela. «Et l'enseignant l'a dit. »

En fait, en tout cas en norvégien, le coordonnant apparait hors du cadre des syntagmes susceptibles d'amener le V2, comme en atteste le fait qu'il peut précéder le sujet préverbal en $(2 b)$, dans une langue où une seule projection pertinente peut se trouver avant le verbe. Donc, ou bien les configurations en (1a-c) ne sont pas du V2, expliquant leur absence en norvégien, ou bien elles en sont, et il manque quelque chose au V2 norvégien. L'analyse choisie aura quelque impact pour l'idée que le $\mathrm{V} 2 \mathrm{du}$ français ancien serait comparable à celui des langues 
scandinaves (voir par exemple Labelle \& Hirschbühler 2019).

L'article s'organise en trois temps. Premièrement, nous considérons les études sur la configuration à sujet postposé avec coordonnant initial en français ancien. Ensuite, le rapport entre les attentes soulevées par ces études et le comportement effectif de la configuration dans le corpus calibré FLAN+ est évalué. En particulier, au vu des données d'observation, est traitée la question de l'analyse syntaxique. En fin de compte, l'analyse à retenir est que la configuration ne relève pas du V2, parce que le verbe ne figure pas en position syntaxique haute. Il s'agit plutôt d'une coordination asymétrique, qui est généralement possible en français ancien, mais non en français actuel. Les résultats sont résumés dans la section conclusive.

\section{[2] COORDONNANTS ET POSTPOSITION DU SUJET}

Cette section passe en revue quelques-unes des analyses dont a fait l'objet la configuration à l'étude Coordonnant + verbe + sujet exprimé illustrée en (1)-(3) en français ancien.

A la suite de Lemieux (1992), Barbara Vance (1993) considère le rôle de et introduisant une proposition en français ancien, à la fois à la jonction de deux propositions comme en (1a) et à l'initiale d'une phrase comme en (1b). La raison de s'intéresser à ces cas est qu'ils reposent la question du statut du sujet dont on sait qu'il évolue dans l'histoire de la langue. A considérer la phrase suivante tirée du texte en prose Queste del Saint Graal auquel réfère Vance,

$$
\begin{aligned}
& \text { Et ele descent et vient devant le roi } \\
& \text { Et elle descend et vient devant le roi } \\
& \text { «Et elle descend et vient devant le roi » }
\end{aligned}
$$

on peut se demander si dans la deuxième séquence on est face à la coordination des groupes verbaux avec mise en commun du sujet, ou à celle de deux propositions avec dans la seconde un sujet non-exprimé. La réponse à cette question a un impact sur la compréhension du devenir du sujet non-exprimé, et sur son évaluation quantitative (Prévost 2015, Marchello-Nizia 2017, Capin \& Larrivée 2020). Une double analyse n'est pas toujours possible, cependant, quand le parallélisme n'est plus manifeste entre les propositions conjointes, remettant en doute la possible coordination du seul groupe verbal. Dans le cas suivant,

(4) lors entra en la sale a cheval une molt bele damoisele; alors entra dans la salle à cheval une très belle jeune-femme 
et fu venue si grant oirre que... et fut venue si grande vitesse que " Alors entra dans la salle une belle jeune femme à cheval et elle était venue si vite que ...»

Vance estime que « the constructions are not parallel, the preverbal constituent of the first clause does not distribute over the second clause, and the tenses and persons of the verb are not necessarily the same inthe two clauses ${ }^{1}$. » $(1993: 287)$. L'analyse d'une coordination des groupes verbaux est impossible quand l'antécédent du sujet de la deuxième proposition n'est pas le sujet de la première proposition à laquelle il est coordonné, comme on le voit ci-dessous par des exemples de la même Queste (avec coordination par ne en $(5 \mathrm{~b})$ - sur ne, voir Ingham 2014).

(5) a. si dient que il est a lor avis li plus biaus ... et fleroit autresint soif si dirent que il est à leur avis le plus beau ... et sentait très bon « Ils dirent que c'est à leur avis le plus beau ... et il sentait merveilleusement bon »

b. Sire, vos avez grant piece chevauchié toz seuls et je toz seuls, Sire, vous avez beaucoup chevauché tout seul et moi tout seul ne n'avons riens trod. ni n'avons rien trouvé "Sire, vous avez beaucoup chevauché tout seul, et moi tout seul aussi, et nous n'avons rien trouvé. »

De nécessité, ces exemples mettent en jeu la coordination de deux propositions, avec un sujet nul dans la seconde, qui serait bel et bien irrecevable en français actuel,

(6) *Vous avez tant cherché, et n'avons rien trouvé.

où le sujet nul n'est globalement pas disponible comme option productive. Cependant, ce ne sont pas tous les sujets postposés qui sont possibles avec un coordonnant initial. En effet, seuls les sujets nominaux sont attestés, jamais les sujets clitiques $^{2}$. Les clitiques qui se retrouvent avec un coordonnant initial sont ceux qui précédent le verbe, comme en (3). De cette restriction aux sujets nominaux ${ }^{3}$,

[1] «Les constructions ne sont pas parallèles, le constituant préverbal de la première proposition ne s'applique pas à la seconde, et les temps et personnes du verbe ne sont pas nécessairement les mêmes dans les deux propositions. "

[2] Je maintiens par commodité ce terme qui est possiblement un anachronisme, selon la chronologie qu'on envisage pour la transformation en clitique des pronoms sujets.

[3] Un relecteur souligne avec justesse que la raison pour laquelle la position basse du verbe excluerait les 
Vance en déduit que la coordination s'applique au niveau inférieur du syntagme inflexionnel (I’), plutôt qu'en une place du fondement plus élevée.

$$
\text { et [IP V [VP SUJET [V' t ...]]] }
$$

Préservant le parallélisme entre sujet exprimé (7) et sujet nul (8)

$$
\text { et [IP V [VP pro [V't ...]]] }
$$

cette structure voit le sujet rester dans sa position d'origine. Parce que le verbe ne monte pas en place du fondement, il ne précède pas les clitiques, lesquels ne se retrouvent qu'en position préverbale dans cette configuration. La séquence Coordonnant + verbe + sujet a donc une syntaxe différente de la construction V2 où le verbe et les éléments qui le précèdent se situent en place du fondement. Considérez les deux exemple suivants du plus ancien texte légal en français, les Lois de Guillaume de 1150.
a. e par le dener que li sire
durrad: si erent quite

et par le denier que le seigneur donnera si seront quitte

cil ki meindrunt en sun demeine

ceux qui resteront en son domaine

« Et par le denier que donnera le seigneur alors seront quitte ceux qui demeurent en son domaine "

b. e sil nel ad: dunc numerad il sun heimelborch

Et s'il ne l'a donc nommera il son heimelborch

«Et s'il ne l'a pas donc nommera-t-il son heimelborch»

En (9a), le coordonnant doit se situer au-dessus d'une position topique exprimée par par le denier que li sire durrad, et de la position focale où se trouverait si (Ledgeway 2008, Wolfe 2018), et du verbe au-dessus du sujet ; de même qu'en (9b), il doit se situer au-dessus de la subordonnée topique (par contraste avec la subordonnée de même type dans la proposition antécédente), du donc qui pourrait occuper la place élevée de $s i$, et du verbe qui précède le sujet clitique. L'exemple (9b) pourrait ainsi être représenté de la façon suivante en partant de la représentation de Vance.

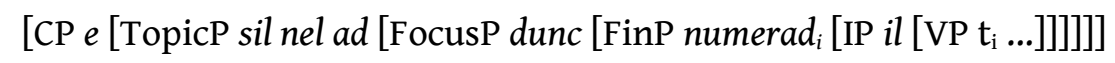

sujets clitiques n'est pas explicitée par Vance. Le raisonnement sous-jacent me semble être le suivant. Il est vrai qu'a priori le groupe nominal comme le clitique sujet sont générés après le verbe dont ils sont l'argument interne. La différence est que l'association du clitique sujet à IP l'oblige à monter, ce qui n'est pas forcément le cas pour le groupe nominal. Ce n'est donc que ces seconds qui peuvent suivre un verbe en position basse. 
Dans ces cas, le coordonnant est extérieur à la proposition, et ne joue pas de rôle formel dans l'inversion du sujet.

Cette analyse est critiquée par Willis (1997). Il fait valoir qu'en (1), si elle s'applique à un groupe inflexionnel (IP) tel qu'en (7), la coordination réunit des termes différents. La coordination de (1a) met effectivement en rapport une proposition complète (Il fut jugié) et une proposition amputée des projections apparaissant en place du fondement. Cela semble contredire le principe selon lequel deux séquences coordonnées soient de même statut. Ce principe explique, hors effet littéraire de zeugme, la curiosité qu'ont pour le lecteur actuel les séquences suivantes,

a. ??Ce sont de sages personnes et intègres.

b. ??Elle a servi le prince et loyalement.

car elles mettent en parallèle une proposition et un circonstant, un groupe nominal et un adjectif. Une façon de réconcilier à cette exigence l'analyse des cas de V2 et de la configuration à l'étude est de produire une analyse parallèle de notre configuration en ( $1 \mathrm{a}-\mathrm{c}$ ) et des cas V2 classiques impliquant la place du fondement comme en (9a-b), ces derniers étant alors analysés comme ayant la structure en (10). Dans les deux cas, l'éventuel coordonnant se retrouverait au-dessus de la place du fondement. Cette position ne serait pas due à la montée du verbe dans cette position de fondement, mais à la présence d'un opérateur de topique nul, dans la même position haute où se trouverait le topique sil nel ad en (10). Ainsi, notre configuration s'analyserait comme Et + Topique + Verbe + Sujet.

Cette analyse avec topique nul, adoptée par Jouitteau (2019) pour rendre compte de différentes constructions à travers les langues, pose plusieurs questions. D'une part, la motivation d'un tel topique pour le cas qui nous intéresse n'est pas manifeste. On voudra bien admettre qu'elle relie la proposition au contexte antécédent, mais toutes les phrases ne sont-elles pas ainsi liées ? Partant, comment reconnaitre la présences de ce topique ? En outre, toujours pour notre configuration, puisque le verbe peut monter en place du fondement avec un topique exprimé, pourquoi ne le pourrait-il pas avec des sujets clitiques postposés? Enfin, s'il y a un topique nul, des phrases avec verbe en position initiale devraient être possibles en français ; or, contrairement au portugais ancien par exemple (Martins 2019), la structure V1 est extrêmement rare dans les textes en prose du français ancien ${ }^{4}$. On est donc face à un dilemme, celui de traiter nos configurations comme des coordinations entre segments de statut différent, enfreignant un principe général, ou comme des coordination symétriques, faisant

[4] Et se limite essentiellement à la proposition hypothétique (qu'a conservée le français dans Soit un segment de droite). Voir la discussion récente dans Klævik-Pettersen (2019). 
des prédictions inexactes.

Le dilemme de la symétrie se reporte au statut du coordonnant lui-même. On se trouve face au choix entre une analyse unique, ou duelle, d'un élément superficiellement identique. C'est le propos de Zimmermann \& Kaiser (2010). On pourrait par exemple estimer que et et consorts sont des coordonnants quand ils sont extérieurs à une proposition qu'il mettent en parallèle avec une autre

(12) a. L'échiquier rendit jugement et Alice obtint la possession Alice demanda la possession et elle l'obtint.

b. Se aucun demande la sesine a son anceisor et len dit encontre Si quelqu'un demande la saisine à son ancêtre et l'on dit encontre que encore vit celui de qui il demande la sesine que encore vit celui de qui il demande la saisine sa mort doit estre provee (1300. Grand coutumier) sa mort doit être prouvée "Si quelqu'un demande la possession des biens de son ancêtre et que l'on affirme que celui-ci est encore vivant, sa mort doit être prouvée »

et qu'ils sont des adverbes quand ils s'associent à la postposition du sujet dans notre configuration.

a. il fu jugié en l'eschequier que cil jugemenz estoit faus, il fut jugé en le parlement que ce jugment était faux et ot Aales la sèsine (1207. Arrêt de l'échiquier de Normandie) et eut Aales la saisine « et Aales en eut la possession "

b. Et ne n'ot le Roy noient (1279. Enquête Pont Audemer) et ni n'eut le roi rien «Et le Roy n'eut rien»

Ce double statut s'appliquerait à la même forme et, mais aussi aux autres coordonnants ne et, très rarement il est vrai, ou, ce qui semble stipulatif. En outre, la question se pose du statut de l'item quand il se retrouve en tête de phrase, comme en (3), et en (5a), ou encore dans l'exemple suivant.

a. Et se ie ne puis tout faire . aucune chose en feray ie «Et si je ne peux tout faire, je ferai tout de même quelque chose. »

(1300. Grand coutumier)

Cet emploi est extrêmement fréquent en français ancien (Capin \& Badiou- 
Monferran 2020, Capin en préparation), et dans un texte comme le Grand coutumier de Normandie, rend compte d'environ neuf-dixième des usages de et.

S'agit-il alors du coordonnant ou de l'adverbe? Coordonnant, il ne coordonne formellement rien, puisqu'il ouvre la phrase ; adverbe, se pose la question de sa contribution sémantique. Serbat (1990) fait valoir que dans ces emplois, et " sert de tremplin au départ d'un nouvel acte d'énonciation. " A la fois Vance (1993) et Zimmermann \& Kaiser (2010) reprennent la proposition selon laquelle et aurait un contenu sémantique comparable à si. Cette comparaison peut être interprétée soit dans le sens de dire que et signifie et alors, comme si qui peut avoir une valeur temporelle, soit que ce serait un explétif, inséré comme élément de dernier recours (Ledgeway 2008). Les arguments concluants manquant, Zimmermann \& Kaiser (2010) arguent que et est un coordonnant.

Les incertitudes quant aux traitements appellent un sondage empirique. S'il était vrai que les types de sujets dans les structures $E t+$ Verbe + Sujet sont restreints aux groupes nominaux, il pourrait se faire qu'on soit face à une structure qui n'est pas véritablement du V2. C'est ce qu'explore la prochaine section.

\section{[3] COORDONNANTS ET POSTPOSITION DU SUJET DANS UN CORPUS CALIBRÉ}

Ainsi que l'a noté Hans Petter Helland (2006 : 286), une grande diversité caractérise l'usage des coordonnants. La configuration considérée est dans cette section envisagée à la lumière de son comportement dans un corpus calibré. Sa chronologie et sa distribution textuelle sont d'abord envisagées.

\section{[3.1] Chronologie et distribution textuelle}

La configuration qui nous intéresse, avec coordonnant initial et inversion du sujet, est bien attestée, avec une chronologie qui reste à pleinement apprécier. Présente dans les premiers textes en prose comme en attestent les exemples du début du $13^{\mathrm{e}}$ siècle cités ici (par exemple (1a)), elle fait sentir sa présence jusqu'au $17^{\mathrm{e}}$ siècle, bien qu'à cette époque, elle soit déjà archaïque selon Ayres-Bennett (1983 : 116). Dans le corpus ConDE ${ }^{5}$ qui réunit des textes du même type textuel coutumier et de la même région normande, en parcourant l'annotation produite manuellement de 300 propositions de chacun, on établit que la construction était encore survivante au $16^{\mathrm{e}}$ - le coutumier commenté de Le Rouillé (1534) en contenant 5 occurrences, relevant de deux patrons syntaxiques, sur un totalde $20 \mathrm{~V} 2$ (dont 10 productifs)

[5] Constitué dans le cadre du projet RIN éponyme et généreusement soutenu par la Région Normandie. 
a. Et doibt len fuppofer que ... et doit l'on supposer que «Et l'on doit supposer que ...»

b. et fera ledict fergent affignation au feigneur es pletz et fera ledit sergent assignation au seigneur aux plaids ou affifes royaulx/pour ploceder fus ladicte deliurance ou assises royauls/pour procéder sur ladite délivrance « et ledit sergent fera assignation au seigneur en procès ou en assises royales pour procéder à cette décision »

- mais guère au-delà, le Bérault (1614) en contenant une seule,

(16) \& eft cet arreft affiché en tableau tant au Bailliage qu'en la Viconté de Rouen

«Et cet arrêt est affiché en tableau tant au baillage que dans le vicomté de Rouen. »

et Merville (1731) aucune.

Un corpus constitué pour la période antérieure permet de mesurer la vitalité de la construction avant 1450. Il s'agit du corpus FLAN+ (Larrivée 2019), qui couvre la période comprise entre 1150 et 1475 . Il est composé de textes en prose de Normandie, une région où la production écrite précoce est relativement abondante, située entre la zone anglo-normande, où la plupart des premiers matériaux ont été produits, et la zone parisienne, où sera définie la référence normative des usages français. Il est homogène en termes de genre, relatif au domaine juridique, et est équilibré pour trois types de textes juridiques, les coutumiers, les audiences et les enquêtes judiciaires, qui permettent on le verra les comparaisons. Le choix du genre légal s'explique d'une part par sa représentation dans la plupart des régions, permettant les éventuelles comparaisons, par son absence de préoccupations esthétiques qui rapproche de la documentation du changement effectif, et par les chronologies plus précoces qu'il fournit des processus de changement (Capin \& Larrivée 2020 et références incluses). Chacun des 40 et quelque textes est annoté manuellement jusqu'à un maximum de 300 propositions afin de repérer en particulier l'expression et la position du sujet.

Les postposition du sujet sont décomptées. Des exemples typiques en sont donnés ci-après.

(17) a. sin ert feite la iustise de larrun

Si+en sera faite la justice de voleur

«Ainsi la justice contre le voleur sera faite» (Leis Will, 1150)

b. En cest cas ni doit li évesques nului recevoir, (Patronage, 1205) 
En ce cas n'y doit le évesque nul recevoir «En ce cas l'évêque ne doit recevoir personne»

c. et ot Aales la sèsine (Arrêt de l'échiquier de Normandie, 1207-1211) et eut Aales la saisine " et Aales en eut la possession »

De ces décomptes sont exclus les cas formulaires, où la construction est instanciée par une séquence plus ou moins figée, comme par exemple la séquence « et doit len » ci-dessus en (15a). En tenant compte des usages productifs de la construction qui contiennent un seul syntagme initial, que ce soit une particule comme si en (17a), un complément (17b) ou un coordonnant (17c), on obtient les chiffres que donnent le tableau suivant (Larrivée 2021).

\begin{tabular}{|c|c|c|c|c|c|c|c|c|c|}
\hline & \multicolumn{2}{|c|}{$\begin{array}{l}\text { V2 produc- } \\
\text { tif avec } \\
\text { complément } \\
\text { comme seul } \\
\text { syntagme } \\
\text { préverbal }\end{array}$} & \multicolumn{2}{|c|}{$\begin{array}{l}\text { V2 productif } \\
\text { avec parti- } \\
\text { cule comme } \\
\text { seul syn- } \\
\text { tagme pré- } \\
\text { verbal }\end{array}$} & \multicolumn{2}{|c|}{$\begin{array}{l}\text { V2 productif } \\
\text { avec coordon- } \\
\text { nant comme } \\
\text { seul syntagme } \\
\text { préverbal }\end{array}$} & \multicolumn{2}{|c|}{$\begin{array}{l}\text { Total V2 } \\
\text { produc- } \\
\text { tif }\end{array}$} & \multirow[t]{2}{*}{$\begin{array}{l}\text { Totaux } \\
\text { proposi- } \\
\text { tions }\end{array}$} \\
\hline & $\begin{array}{l}\mathbf{n} \\
\end{array}$ & $\%$ & $\begin{array}{r}\mathbf{n} \\
.\end{array}$ & $\%$ & n. & $\%$ & n. & $\%$ & \\
\hline $\begin{array}{l}1150- \\
1249\end{array}$ & 11 & 31 & 5 & 14 & 1 & 3 & 36 & 2,4 & 1526 \\
\hline $\begin{array}{l}1250- \\
1349\end{array}$ & 29 & 25 & 13 & 11 & 32 & 27 & 117 & 3,6 & 3275 \\
\hline $\begin{array}{l}1350- \\
1475\end{array}$ & 14 & 19 & 3 & 4 & 41 & 57 & 72 & 2,1 & 3395 \\
\hline $\begin{array}{l}\text { To- } \\
\text { taux }\end{array}$ & 54 & 24 & 21 & 43,2 & 74 & 32,8 & 225 & 2,8 & 8196 \\
\hline \multicolumn{10}{|c|}{ TABLEAU 1.} \\
\hline
\end{tabular}

On constate que les postpositions du sujet avec le coordonnant sont rares avant 1250. De 1250 à 1350, elles commencent à être bien représentées, avec $27 \%$ de tous les cas. Elles sont dominantes de 1350 à 1475 où elles correspondent à $57 \%$ des cas. Cette dominance s'explique aussi en partie par le fait que les constructions strictes, introduites par un seul syntagme initial, sont prépondérantes dans 
cette dernière période (à $80,5 \%, 58$ des 72 occurrences totales) alors qu'elles sont dominantes dans la deuxième période $(63,2 \%, 74$ de 117$)$ et tout juste minoritaires dans la première $(47,2 \%, 17$ sur 36$)$. En se reportant aux données des coutumiers tardifs, on a donc une chronologie de 1250 à 1550 pour l'usage récurrent de la configuration, et de 1150 à 1650 pour ses manifestations.

Ce que montre également le tableau est que la configuration n'est pas un phénomène propre aux textes de type littéraire généralement utilisés par les diachroniciens dont Vance, puisqu'elle se retrouve dans des textes légaux, et avec une chronologie semblable (sur la dominance des coordination dans la période tardive dans les textes littéraires, voir Dupuis et al 1992). Je me suis posé la question, à laquelle le corpus FLAN+ permet de répondre, de savoir si la configuration appartenait à un type de texte légal particulier. Le relevé de tous les cas, formulaire ou non, est fourni dans ce second tableau.

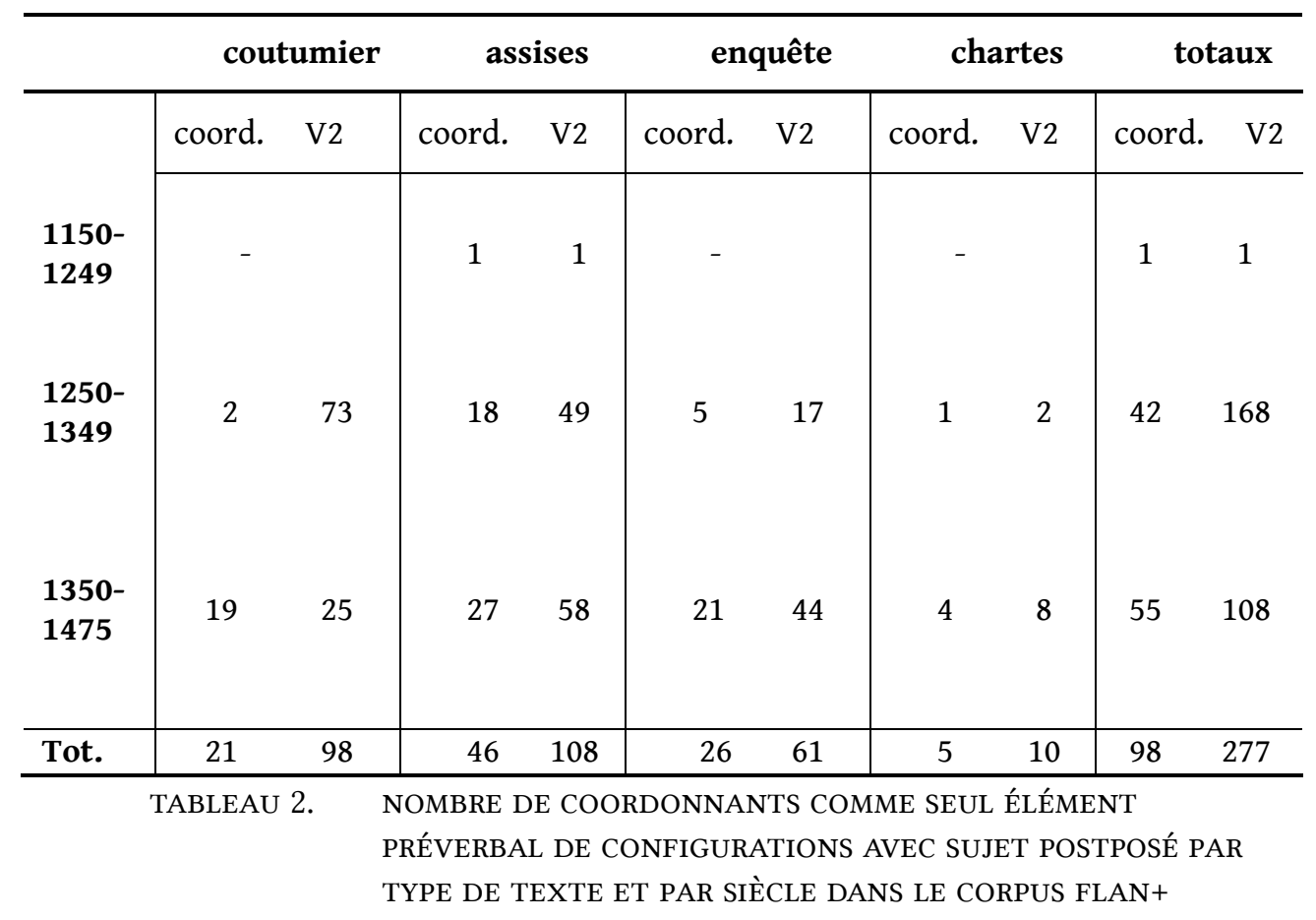

Les données montrent que s'il y a une variation à travers le temps, des enquêtes et assises dans la deuxième période, aux coutumiers et assises dans la troisième, chaque type est susceptible d'attester la configuration.

Une question importante à laquelle on ne peut fournir que des indications ici est celle du registre de la configuration. On pourrait croire que c'est à l'écrit qu'est représenté la postposition du sujet avec un coordonnant initial, et que ce 
phénomène serait absent de l'oral. Evidemment, nous n'avons de l'oral que des représentations textuelles. J'ai considéré deux types de sources. D'une part, je me suis attardé à la correspondance de la famille d'Estouteville (disponible en ligne dans le corpus EPELE, https://www.unicaen.fr/epele/accueil). Cet ensemble allant de la période 1480 à 1520 réunit non seulement des échanges entre nobles, mais aussi avec leurs employés, et certaines pièces entre employés. L'annotation de 501 propositions de lettres représentant ces différentes cas de figure montre que la configuration $\mathrm{Et}+$ verbe + sujet est dominante ( 7 occurrences sur 11 postpositions) ; mais comme les V2 sont le fait exclusif des lettres écrites à des nobles, la dominance n'est pas concluante. Par ailleurs, j'ai considéré les données d'oral représenté que j'ai pu repérer pour le 14 e siècle dans les textes légaux normands. Ont pu être réunis des Actes du fonds de Barbery en français avant 1340, les Registre de plaids de La Ferté en Ponthieu, et des Lettres de rémission, aimablement mis à disposition par l'historien Adrien Dubois, et des passages de Atirement et jugiés d'eschequiers. Les 146 propositions assertives analysées fournissent 4 cas de postposition du sujet. Aucun ne fournit la configuration à l'étude, à une époque où dans le corpus FLAN+, elle est est dominante. S'il est difficile de trancher sur une base aussi ténue, néanmoins, la configuration pourrait bien être un fait de l'écrit. Elle serait alors à rapprocher des phénomènes de démarcation graphique (voir Capin En préparation).

\section{[3.2] Analyse structurelle}

Tournons-nous maintenant vers l'analyse structurelle de la construction. Les données attestées du corpus FLAN+ confirment ce qu'avait constaté Vance. Le sujet postposé est toujours un groupe nominal, comme on le voit dans les exemples (1a-1c). En cela, le français se démarque par exemple de l'italien qui admet en postposition à la fois des sujets nominaux mais aussi des clitiques.

$$
\begin{aligned}
& \text { e cadde ella di sotto, } \\
& \text { et tomba elle de sous } \\
& \text { " et elle tomba par terre "(Poletto 2014, p. 22) }
\end{aligned}
$$

Les clitiques ne sont jamais représentés dans cette construction en français, à l'exception, comme l'avait également noté Vance, de l'on, écrit l'en, et on, dans 4 et 3 occurrences respectivement sur les 98 recensées (y compris les occurrences formulaires) dans FLAN+.

(19) a. et ira l'en en avant a accorder le jugement o discretion «Et l'on accordera le jugement à discrétion » (1386. Ordonnances)

b. Et ne pescheroit on point n'a verge n'a lingne, se monsegneur vouloit 
«Et on ne pêcherait pas ni avec une canne ni avec une ligne, si Monseigneur le demandait » (1380. Coustumes de l'eau de Saine)

c. et ne sera l'en plus tenu à prendre le dit orge ou seigle après le dit terme passé.

" et l'on ne sera plus tenu de prendre l'orge ou le seigle après la date dite. » (1450. Enquête)

Cinq d'entre elles sont formulaires, (19a) apparaissant 2 fois dans le même texte et la séquence « doit l'en/on savoir » en (20) apparaissant 3 fois.

(20) et doit l'en savoir que les tonneaus desus dis doivent estre contez as personnes à qui l'en doit les moeisons (1291. Vicompté de l'eau de Rouen)

" et l'on doit savoir que ces tonneaux doivent être mis au compte des personne à qui l'on doit les moissons »

L'attestation en (19b) est remarquable, car elle contient une négation, qui est rare ; celle-ci ne concerne que 8 occurrences, apparait avec 3 occurrences avec sujet nominal, 3 avec l'en/on, et 2 avec nul.

Le ne de négation apparaissant en position préverbale est accompagné d'autres clitiques compléments, et ci-contre les douze séquences recensées.

(21) a. et le prist li dit Guillaume u non du dit Juliot Bouvet « et le dit Guillaume le prit au nom de ce Juliot Bouvet "

b. et en eut le dit Guillaume VI lb " et le dit Guillaume en eu 4 livres »

c. et la doit le frère touz jours porte sur soi tant comme il vivra " et le frère doit toujours la porter sur lui toute sa vie "

d. et les pourcachera ladite sous aage a ses cous " et cette fille mineure les poursuivra à ses coûts "

e. et s'en est mis lidis Jehan en l'eformacion " et ce Jean en a été mis en information"

f. et le somma lidis Loeis de faire presentement ledit compte " et ce Louis le somma de faire ce compte immédiatement "

g. et se tint Jehan de Haravesnes pour adjournés comme procureur «Et Jean de Haravesnes se considéra comme ajourné en tant que procureur"

h. Et s'obligerent lesdis prevost et eschevin que ... «Et ces prévôts et échevins se firent l'obligation que »

i. et en est deu a monsgr obéissance de li présenter cescun an trois des bourgois de la dite ville « et le droit de lui présenter chaque année trois des bourgeois de cette 
ville en est due à Monseigneur »

j. et en rapportera $G$. la chose qu'il demande " et G. en rapportera la chose qu'il demande »

k. mais se paiera par chacune partie sa porcion " mais sa portion sera payée par chaque partie»

1. et luy racomptoit l'ange la pitié qui estoit eu royaulme de France " et l'ange lui racontait la pitié qui était au royaume de France »

Donc, le verbe, dans cette construction, se situe au-dessus du sujet nominal et de pronoms comme nul et l'on dans la structure hiérarchique de la phrase. Ce sujet serait en position de base, à l'intérieur du groupe verbal. En tant qu'argument interne, il serait directement adjoint au verbe, précèdant les compléments. C'est le cas en (1a) et (1b), et en (21a), (21j) et (21l), même si (21i) et (21k) ne vont pas dans ce sens, peut-être à la suite d'un scrambling. On constate en outre l'absence de sujet nominal entre le participe et le verbe en (21e) et (21i), indiquant l'absence de mouvement ${ }^{6}$. Si le sujet ne bouge pas de sa position de base, le verbe ne monte pas au-dessus de la position occupée par les clitiques, complément, ne de négation ou sujet (Labelle \& Hirschbühler 2005). Ainsi, puisque le verbe ne monte pas en place du fondement, cette configuration n'est pas une construction V2. Il en découle que le et devrait se situer immédiatement au-dessus du verbe, dans une position relativement basse comme en (7).

$$
\text { et [IP V [VP SUJET [V't ...]]] }
$$

Mais qu'en est-il de l'objection de Willis ? Quand le coordonnant amenant un sujet postposé relie à une proposition antécédente comme en (1a),

(23) il fu jugié en l'eschequier que cil jugemenz estoit faus, il fut jugé en le parlement que ce jugment était faux et ot Aales la sèsine (1207. Arrêt de l'échiquier de Normandie) et eut Aales la saisine « et Aales en eut la possession »

il conjoint deux unités de nature différente. Si cela était légitime, on s'attendrait à trouver des coordination entre unités de nature différente comme dans les

[6] Un relecteur note que « il s'agit de la voix pronominale en (21e) et la voix passive dans (21i) (où le sujet est d'ailleurs aussi extrêment lourd et peut-être déplacé en fin de phrase pour des raisons prosodiques/de rythme) ; même dans les propositions V2, le sujet se trouve souvent après le participe passé avec des verbes inaccusatifs ou à la voix passive (ce que Vance (1997) nommme 'discontinuous inversion'). Il faudrait donc trouver des exemples avec un verbe transitif, et de préférence un sujet moins lourd qu'en (21i) pour que cette configuration soit un indice fiable du non-mouvement du verbe. » De tels exemples seraient fort utiles, mais demeurent difficiles à trouver à une époque où le passé composé n'est pas pleinement représenté, et leur identification doit être espérée dans des travaux ultérieurs. 
élicitations suivantes.

(24) a. ?? Ce sont de sages personnes et intègres.

b. ?? Elle a servi le prince et loyalement.

Et bien entendu, de telles séquences, curieuses en français actuel, sont attestées à date ancienne

(25) a. Cist ert sis niez qui feelment les a serviz et lealment. Ce sera ce neveu qui fidèlement les a servi et loyalement "Ce sera ce neveu qui fidèlement les a servis, et loyalement"

(Le roman du Mont Saint-Michel)

b. Les iuges sunt sages persones \& autentiques Les juges sont sages personnes et authentiques «Les juges sont des personnes sages et intègres "

(1300. Grand coutumier)

c. ce que la gregnor partie \& la plus sage iugera ce que la grande partie et la plus sage jugera "Ce que la plus grande et plus sage partie jugera »

(1300. Grand coutumier)

La chose avait déjà été notée par Nathalie Fournier qui cite Corneille (1998 ; voir aussi Capin \& Badiou-Monferran 2020).

(26) Albe le veut et Rome.

Les coordinations asymétriques semblent donc acceptables en français ancien. C'est parce que le français ancien les accepte qu'en (1a) on peut envisager la coordination entre une proposition complète et la structure plus limitée représentée par la seconde proposition.

Ces faits établis, nous pouvons résumer le propos dans la conclusion.

\section{[4] COORDONNANTS ET POSTPOSITION DU SUJET}

Le but de ce travail est de réévaluer la construction à inversion du sujet introduite par un coordonnant en français ancien, illustrée dans les exemples (1a-c). La question que cette construction pose est de savoir si elle relève de la structure à verbe en seconde position (V2). Vance fait valoir que le sujet postposé n'est jamais qu'un groupe nominal, et que le clitique ne peut que précéder le verbe ; cela suppose que le verbe jamais suivi d'un clitique se trouve en position syntaxique relativement basse, et non pas en place du fondement comme dans la construction V2. Willis objecte que cette analyse équivaut à une coordination 
asymétrique en (1a), entre la structure plus limitée représentée par la seconde proposition et la proposition complète à laquelle cette structure est coordonnée ; une coordination symétrique force à adopter une analyse V2. L'analyse a in fine un impact sur la façon d'envisager l'item initial, coordonnant ou autre adverbe. C'est l'analyse de Vance selon laquelle la construction considérée ici voit le verbe se situer en position syntaxique basse que valident les données calibrées examinées. La construction, dominante pour les cas d'inversion du sujet entre 1350 et 1475 , et attestée de 1150 à 1650 , ne présente que des sujets nominaux, généralement en proximité immédiate avec le verbe, mais jamais de clitiques. En cela, les données de notre corpus concordent avec des données littéraires des études antécédentes, ce qui est en soi un résultat remarquable. L'objection d'une coordination asymétrique tombe, parce que le français ancien admet ces coordinations non seulement pour les proposition à structure limitée, mais aussi pour des constituants comme le sujet, les adjectifs et les adverbiaux. Il n'y a donc pas lieu de chercher au coordonnant un statut autre.

Cette analyse rend compte des données observées du français ancien dans un corpus calibré de textes du même type et de la même région. Elle explique que la même structure ne se retrouve pas en norvégien, puisque cette structure n'est pas du V2. Mais on pourrait estimer que dans une structure où la proposition n'est pas coordonnée à une première comme en (1b), l'analyse V2 reste possible, et cette analyse doit de toute façon s'appliquer à l'italien. Comme tous les classiques, la question sera certainement à réévaluer.

\section{REMERCIEMENTS}

Je veux remercier les éditeurs de l'occasion qu'ils me donnent de présenter mes hommages à Hans Petter. Hormis les commentaires des éditeurs et des relecteurs, et celui de participants à différents événements de recherche où le travail a été présenté, une version initiale de ce travail a bénéficié des sagaces observations de Daniéla Capin, et les erreurs restantes sont de mon fait. Je reconnais avec gratitude le généreux financement que la Région Normandie a accordé au projet CONDE ayant permis le développement de certaines des ressources textuelles utilisées dans ce travail.

\section{REFERENCES}

Antoine, Gérald. 1958-1962. La Coordination en français, 2 t. d'Artrey.

Ayres-Bennett, Wendy. 1983. Vaugelas and the development of the French language: theory and practice. Oxford University Press.

Baulier, F. 1956. Contribution à l'étude de l'inversion du sujet après la 
conjonction "et". Le français moderne 24. 249-257.

Bergh, L. 1952. Quelques réflexions sur l'inversion après la conjonction et en ancien et en moyen français. Mélanges de philologie romane offerts à M. Karl Michaëlsson par ses amis et ses élèves, 43-55. Bergendahl.

Capin, Daniéla. En préparation. Coordination, complémentation et sujet nul : ce que la Coutume de Normandie nous dit. Studia Linguistica Romanica.

Capin, Daniéla \& Claire Badiou-Monferran. 2020. À l'aune de la transcatégorisation : modélisation des emplois de et dans la langue d'hier et d'aujourd'hui. D. Th. Do-Hurinville et alii (dirs). Transcatégorialité et transcatégorisation. Société de Linguistique de Paris. 153-194. Société de Linguistique de Paris.

Capin, Daniéla \& Pierre Larrivée. 2020. La continuité référentielle du sujet nul dans les textes législatifs en français ancien. Estèle Dupuy, Victor Millogo \& Marie-Helène Lay (dirs). La continuité référentielle ou le choix des mots, 147-164. Presses universitaires de Rennes.

Dupuis, Fernande, Monique Lemieux \& D. Gosselin. (1992). Conséquences de la sous-spécification des traits de Agr dans l'identification de Pro. Language Variation and Change 3. 275-299.

Fournier, Nathalie. 1998. Grammaire du français classique. Belin.

Helland, Hans Petter. 2006. Ny fransk grammatikk: morfologi, syntaks og semantikk. Universitetsforlaget.

Ingham, Richard. 2014. Old French negation, the Tobler/Mussafia Law, and V2. Lingua 147. 25-39.

Jouitteau, Mélanie. 2019. Embedded V2 is syntactically integrated. Présentation à la 52e rencontre annuelle de la Societas Linguistica Europaea, 21 - 24 août 2019.

Klævik-Pettersen, Espen. 2019. Inversion, $V$-to-C, and verb second: an investigation into the syntax and word order of Old French and Late Latin. Thèse de doctorat, Universitetet i Oslo.

Labelle, Marie \& Paul Hirschbühler. 2018. Topic and focus in Old French V1 and V2 structures. Revue canadienne de linguistique 63(2). 264-287.

Labelle, Marie \& Paul Hirschbühler. 2005. Changes in clausal structure and the position of clitics in Old French. Montserrat Batllori, Maria-Lluisa Hernanz, 
Carme Picallo \& Francesc Roca (eds.), Grammaticalization and parametric variation, 149-178. Oxford University Press.

Larrivée, Pierre. 2019. To be or not to be informational : Preverbal complements in Medieval French V2 configurations. Glossa 4(1). 1-19.

Larrivée, Pierre. 2021. An Information Structure scenario for V2 loss in Medieval French. Diachronica.

Ledgeway, Adam. 2008. Satisfying V2 in early Romance: Merge vs. Move. Journal of Linguistics 44(2). 437-470.

Lemieux, Monique. 1992. Et dans les constructions à sujet nul et à sujet postposé en moyen français.Travaux de linguistique 25. 59-75.

Marchello-Nizia, Christiane. 2017. De S0 à SV: Vers le sujet obligatoire et antéposé en français, les dernières phases d'un changement. Journal of French Language Studies 28(1). 1-19.

Martins, Ana Maria. 2019. Against V2 as a general property of Old Romance languages. Ingo Feldhausen, Martin Elsig, Imme Kuchenbrandt \& Mareike Neuhaus (eds.), Romance Languages and Linguistic Theory 15: Selected papers from 'Going Romance' 30, Frankfurt. Benjamins. 10-33.

Poletto, Cecilia. 2014. Word order in Old Italian. Oxford University Press.

Prévost, Sophie. 2015. Diachronie du français et linguistique de corpus: une approche quantitative renouvelée. Langages 197(1). 23-45.

Serbat, Guy. Et « jonctif » de propositions : une énonciation à double détente. L'Information Grammaticale 46. 26-28.

Skårup, Povl. 1975. Les premières zones de la proposition en ancien français. Essai de syntaxe de position. Etudes romanes de l'université de Copenhague.

Vance, Barbara S. 1993. Verb-first declaratives introduced by et and the position of pro in Old and Middle French. Lingua 89. 281-314.

Willis, David. 1997. Clausal coordination and the loss of verb-second in Welsh. Oxford Working Papers in Linguistics, Philology and Phonetics 2. 151-172.

Wolfe, Sam. 2018. Verb Second in Medieval Romance. Oxford University Press.

Zimmermann, Michael \& Georg A. Kaiser. 2010. Much ado about nothing? On the categorial status of $e t$ and ne in Medieval French. Corpus 9. 265-290. 
COORDONNÉES

Pierre Larrivée

Université de Caen Normandie

pierre.larrivee@unicaen.fr 